\title{
Spectral Functions and Properties of Nuclear Matter
}

\author{
V. A. Danilenko, ${ }^{1}$ K. A. Gridnev, ${ }^{1}$ and A. S. Kondratyev ${ }^{2}$ \\ ${ }^{1}$ Department of Nuclear Physics, Saint Petersburg State University, Neva River Embankment 7/9, \\ Saint Petersburg 199034, Russia \\ ${ }^{2}$ Department of Physics, Herzen State Pedagogical University of Russia, Moika River Embankment 48, \\ Saint Petersburg 191168, Russia \\ Correspondence should be addressed to A. S. Kondratyev; kondrat6125@mail.ru
}

Received 14 April 2013; Accepted 19 June 2013

Academic Editor: Adam Lipowski

Copyright (C) 2013 V. A. Danilenko et al. This is an open access article distributed under the Creative Commons Attribution License, which permits unrestricted use, distribution, and reproduction in any medium, provided the original work is properly cited.

The Green's function method in the Kadanoff-Baym version provides a basic theory for nuclear dynamics which is applicable also to nonzero temperature and to nonequilibrium systems. At the same time, it maintains the basic many-body techniques of the Brueckner theory that makes reasonable a comparison of the numerical results of the two methods for equilibrium systems. The correct approximation to the spectral function which takes into account the widths of energy levels is offered and discussed, and the comparison of the values of binding energy in the two methods is produced.

\section{Introduction}

The only microscopic theory which is capable of describing dynamical and statistical properties of quantum many-body systems in a comprehensive way is the Green's function approach initiated by Martin and Schwinger and later developed by Kadanoff and Baym [1,2] with specific application to nuclear matter in [3-6]. Traditional many-body theories of nuclei such as Brueckner's are based on a quasiparticle picture ab initio neglecting the widths of the energy levels associated with the strong interactions when calculating the binding energy. In its original form, the Brueckner theory only considers particle propagation in intermediate states by defining a two-body reaction matrix $K$ of "effective" interaction or similarly defined objects [3]. Different approximations to the spectral functions in the Kadanoff-Baym method established a link between the Brueckner and the Green's function theories [3-6]. Similar approximations to the spectral functions and kinetic equations were considered also in [7-9]. Unfortunately, all the approximations offered in [3-9] turned to be unsatisfactory as it was shown in [10].

The purpose of this paper is to generalize the results of [10] concerning the spectral function and to clarify some of the quantitative relations between the two methods in nuclear physics on the basis of these results. We show that the self-consistent approximations in the two theories lead to very close results, although numerical calculations of the binding energy are still limited by our limited knowledge of nuclear forces and many-body effects.

\section{Spectral Functions of the Particle and Quasiparticle States in the Kadanoff-Baym Approach}

The KB formalism results in the following general expression for a one-particle spectral function $a(\mathbf{p}, \omega)$ for a system in equilibrium state $[1,2,10]$ :

$$
a(\mathbf{p}, \omega)=\frac{\Gamma(\mathbf{p}, \omega)}{(\omega-e(\mathbf{p}, \omega))^{2}+\Gamma^{2}(\mathbf{p}, \omega) / 4},
$$

where

$$
e(\mathbf{p}, \omega)=E^{\mathrm{HF}}(\mathbf{p})+\operatorname{Re} \Sigma_{c}(\mathbf{p}, \omega),
$$


and $E^{\mathrm{HF}}(\mathbf{p})$ is a one-particle energy in the Hartree-Fock approximation. Real and imaginary $(\Gamma)$ parts of the correlation self-energy function $\Sigma_{c}$ are related through the Hilbert transform

$$
\operatorname{Re} \Sigma_{c}(\mathbf{p}, \omega)=P \int_{-\infty}^{\infty} \frac{d \omega^{\prime}}{2 \pi} \frac{\Gamma\left(\mathbf{p}, \omega^{\prime}\right)}{\omega-\omega^{\prime}} .
$$

Here, $P$ refers to a principal value integration.

The spectral function satisfies the exact sum rule:

$$
\int_{-\infty}^{\infty} \frac{d \omega}{2 \pi} a(\mathbf{p}, \omega)=1
$$

for all values of momentum p. This result follows directly from the commutator relations for field operators and can serve as a keystone for checking all approximations to the spectral function (1).

It was shown in $[1,2]$ that, in the case of slowly varying in space and time disturbances, after the transition to the Wigner coordinates and Fourier transform with respect to the difference of the initial time and space variables, the spectral function obeys $(9-27)$ in $[1,2]((10)$ in [10]) and can be represented by the same equation (1), and only all the quantities entering (1) become also the functions of space and time variables $R$ and T. This property is one more keystone for checking the validity of different approximations to the spectral function.

We consider a system in equilibrium state and define the quasiparticle energy $E(\mathbf{p})$ as a solution of $[1,2]$

$$
E(\mathbf{p})=\left.e(\mathbf{p}, \omega)\right|_{\omega=E(\mathbf{p})} .
$$

In the case of a small width of the energy level, when $\Gamma \rightarrow 0$, we get

$$
a(\mathbf{p}, \omega)=2 \pi \delta(\omega-e(\mathbf{p}, \omega))
$$

where $\delta(x)$ is the Dirac delta function. It is shown in $[1,2]$ that the argument of the delta function is a monotonically increasing function of $\omega$ for all $\mathbf{p}$ near the Fermi level where $\Gamma \rightarrow 0$. It then follows that, in this case for every $\mathbf{p}$, there exists just one root of (5).

Now we expand $e(\mathbf{p}, \omega)$ as a function of $\omega$ in Taylor series near the value $E(\mathbf{p})$ and save only linear terms:

$$
e(\mathbf{p}, \omega)=E(\mathbf{p})+\left.\frac{\partial e(\mathbf{p}, \omega)}{\partial \omega}\right|_{\omega=E(\mathbf{p})}(\omega-E(\mathbf{p})) .
$$

Substituting (7) into (1), we get the formula for $\left.a\right|_{\mathrm{QP}}$ called the spectral function of the quasiparticle state:

$$
a_{\mathrm{QP}}(\mathbf{p}, \omega)=\frac{Z^{2} \Gamma(\mathbf{p}, E(\mathbf{p}))}{(\omega-E(\mathbf{p}))^{2}+Z^{2} \Gamma^{2}(\mathbf{p}, E(\mathbf{p})) / 4},
$$

where $Z=Z(\mathbf{p})$ is a renormalizing factor defined by the expression

$$
Z^{-1}=1-\left.\frac{\partial e(\mathbf{p}, \omega)}{\partial \omega}\right|_{\omega=E(\mathbf{p})}
$$

It is easy to prove that $Z<1$ for all values of $\mathbf{p}$ when correlation energy $\Sigma_{c}$ is taken into account $[1,2]$. The quantity $\gamma=Z \Gamma$ can be considered the width of the quasiparticle state, and (8) can be rewritten as

$$
a_{\mathrm{QP}}(\mathbf{p}, \omega)=Z \frac{\gamma}{(\omega-E(\mathbf{p}))^{2}+\gamma^{2} / 4} .
$$

A severe drawback of $a_{\mathrm{QP}}$ is that it normalizes to $Z$ rather than to 1 as in (4):

$$
\int_{-\infty}^{\infty} \frac{d \omega}{2 \pi} a_{\mathrm{QP}}(\mathbf{p}, \omega)=Z<1 .
$$

This deficiency can be remedied by better approximations to the spectral function (1).

If we neglect the width of the quasiparticle state $\gamma \rightarrow 0$, (10) reads

$$
a_{\mathrm{QP}}(\mathbf{p}, \omega)=2 \pi Z \delta(\omega-E(\mathbf{p}))
$$

with the same normalization (11).

Another deficiency associated with the quasiparticle spectral function (10) is the change of $\omega$ in the width $\Gamma(\mathbf{p}, \omega)$ for $E(\mathbf{p})$, which contradicts the dispersion relation (3). As a result, the following approximations to the spectral function (1) were introduced in [3-9].

Quasiclassical approximation $a_{\mathrm{QC}}[3,4]$ :

$$
a_{\mathrm{QC}}=2 \pi \delta(\omega-E(\mathbf{p})) .
$$

Quasiparticle approximation $a_{\mathrm{QP}}$ in the form (12) [3-5]:

$$
a_{\mathrm{QP}}=2 \pi Z \delta(\omega-E(\mathbf{p})) .
$$

Extended quasiparticle approximation $a_{\mathrm{EQP}}[3-6]$ :

$$
a_{\mathrm{EQP}}(\mathbf{p}, \omega)=2 \pi Z \delta(\omega-E(\mathbf{p}))+P \frac{\Gamma(\mathbf{p}, \omega)}{(\omega-E(\mathbf{p}))^{2}} .
$$

Improved quasiparticle approximation $a_{\mathrm{IQP}}$ was offered in [4] in the form

$$
a_{\mathrm{IQP}}(\mathbf{p}, \omega)=2 \pi Z \delta(\omega-E(\mathbf{p}))+Z P \frac{\Gamma(\mathbf{p}, \omega)}{(\omega-E(\mathbf{p}))^{2}},
$$

but this approximation was never used in numerical calculations.

The approximations (13) and (14) correspond to the cases when the widths of the energy levels are neglected, and the approximation (13) is twice contradictory: the correlation part of interaction is taken into account, but the normalization $Z$ caused by this interaction is neglected. The approximations (15) and (16) correspond to the cases when the widths of the levels are taken into account, but they were obtained by a nonadequate expansion of the expression (1) in powers of $\Gamma$ starting with the Dirac delta function. It was shown in [10] that these approximations do not obey (9-27) in $[1,2]$ in the case of slowly varying disturbances and consequently cannot be correct; as it was stated previously, equilibrium and nonequilibrium approximations are genetically linked with one another $[1,2]$. 
The way of obtaining a correct approximation $a_{i}$ to the spectral function which takes into account the widths of the levels and obeys (10) in [10] can be associated with the integral Fourier transform. Such an approximation looks like

$$
a_{i}(\mathbf{p}, \omega)=2 \pi Z \delta(\omega-E(\mathbf{p}))+Z^{2} P \frac{\Gamma(\mathbf{p}, \omega)}{(\omega-E(\mathbf{p}))^{2}} .
$$

The proof of (17) starts with the formula

$$
\int_{-\infty}^{\infty} d t \exp \left(-\frac{\Gamma}{2}|t|\right) \exp (i t x)=\frac{\Gamma}{x^{2}+\Gamma^{2} / 4}, \quad \Gamma>0 .
$$

We expand the first exponent in the left side of (18) in powers of $\Gamma$ and integrate term by term using the relations [11-13]

$$
\begin{array}{r}
\int_{-\infty}^{\infty}|t|^{2 n+1} \exp (i t x) d t=-2 \sin \left((2 n+1) \frac{\pi}{2}\right) \frac{(2 n+1) !}{|x|^{2 n+2}}, \\
n=0,1,2, \ldots,
\end{array}
$$

$$
\begin{array}{r}
\int_{-\infty}^{\infty} t^{2 n} \exp (i t x) d t=(-i)^{2 n} 2 \pi \delta^{(2 n)}(x), \\
n=0,1,2, \ldots,
\end{array}
$$

where $\delta^{2 n}(x)$ is the $2 n$th derivative of the Dirac delta function. We should mention that the uniform convergence of a series is only a sufficient, but not a necessary, condition for the term-by-term integration validity $[14,15]$. Although the uniform convergence of the Taylor series resulting from the expansion of the exponent in (18) is absent, the term-by-term integration, as we will see, leads to the reasonable result.

The terms of the expansion containing the odd powers of $\Gamma$ in correspondence with (19) generate a geometric sequence with the common ratio $r$ :

$$
r=-\frac{\Gamma^{2}(\mathbf{p}, \omega)}{4(\omega-e(\mathbf{p}, \omega))^{2}} .
$$

The sum of the sequence $a_{\text {odd }}(\mathbf{p}, \omega)$ equals to

$$
a_{\mathrm{odd}}(\mathbf{p}, \omega)=\frac{\Gamma(\mathbf{p}, \omega)}{(\omega-e(\mathbf{p}, \omega))^{2}+\Gamma^{2}(\mathbf{p}, \omega) / 4} .
$$

The expression (22) coincides with the general formula (1) for the spectral function.

The terms of the expansion containing the even powers of $\Gamma$ can be considered on the basis of the formula [15, page 92]

$$
x \delta^{\prime}(x)=-\delta(x) .
$$

It is easy to prove by mathematical induction that

$$
\delta^{(n)}(x)=\frac{(-1)^{n} n !}{x^{n}} \delta(x) .
$$

In correspondence with (20) and (24) the terms even in powers of $\Gamma$ generate a geometric sequence with the same common ratio (21). The sum $a_{\text {even }}(\mathbf{p}, \omega)$ of the sequence is equal to

$$
\begin{aligned}
a_{\text {even }}(\mathbf{p}, \omega)= & 2 \pi \frac{(\omega-e(\mathbf{p}, \omega))^{2}}{(\omega-e(\mathbf{p}, \omega))^{2}+\Gamma^{2}(\mathbf{p}, \omega) / 4} \\
& \times \delta(\omega-e(\mathbf{p}, \omega)) .
\end{aligned}
$$

The contribution of $a_{\text {even }}(\mathbf{p}, \omega)$ to the sum rule (4) is equal to zero:

$$
\int_{-\infty}^{\infty} \frac{d \omega}{2 \pi} a_{\mathrm{even}}(\mathbf{p}, \omega)=0 .
$$

The obtained results prove the validity of the produced expansion and term-by-term integration and testify to the approximation of the spectral function with the precision to the linear in $\Gamma$ terms in the form (17).

\section{Binding Energy of Nuclear Matter}

The Brueckner theory of nuclear matter [16] is based on the matrix $K$ of effective interaction with the diagonal elements defined by formula (1) in [4]. One-particle energies $e_{p}$ entering the expression for the diagonal elements are given by the equation

$$
e_{p}=\frac{p^{2}}{2 m}+\operatorname{Re} V_{B}^{(1)}\left(\mathbf{p}, e_{p}\right) .
$$

The final expression for the quasiparticle energy $\varepsilon_{B}(\mathbf{p})$ in the Brueckner theory looks like $[4,17]$

$$
\varepsilon_{B}(\mathbf{p})=\frac{p^{2}}{2 m}+V_{B}^{(1)}\left(\mathbf{p}, e_{p}\right)+V_{B}^{(2)}\left(\mathbf{p}, e_{p}\right)+V_{B}^{(3)}\left(\mathbf{p}, e_{p}\right) .
$$

The expressions for the potentials $V_{B}^{(1)}\left(\mathbf{p}, e_{p}\right), V_{B}^{(2)}\left(\mathbf{p}, e_{p}\right)$, and $V_{B}^{(3)}\left(\mathbf{p}, e_{p}\right)$ are given correspondingly by the formulas (6), (7), and (10) in [4]. The results of numerical calculations presented in [3-6] were based on these expressions with different approximate transformations. The discussion of these transformations can be found in $[4,6]$.

Numerical calculations on the basis of spectral functions were presented with a comparison of the results obtained through the two approaches, based on the Brueckner theory and on the spectral functions [3-6]. The results obtained with the different methods are as follows [4].

The binding energy $E_{\mathrm{QC}}$ (per nucleon) in the quasiclassical approximation (13) is

$$
E_{\mathrm{QC}}=10.5 \mathrm{MeV} / \text { nucleon. }
$$

The binding energy $E_{\mathrm{QP}}$ in the quasiparticle approximation (14) is

$$
E_{\mathrm{QP}}=15.9 \mathrm{MeV} / \text { nucleon. }
$$

The binding energy $E_{\mathrm{EQP}}$ in the extended quasiparticle approximation (15) is

$$
E_{\mathrm{EQP}}=17.4 \mathrm{MeV} / \text { nucleon. }
$$


In the Brueckner theory, when the formula (27) for the oneparticle energy $e_{p}$ is used in (28) for the quasiparticle energy $\varepsilon_{B}(\mathbf{p})$, the binding energy $E_{B}$ is

$$
E_{B}=16.7 \mathrm{MeV} / \text { nucleon. }
$$

When $e_{p}$ in the formula (28) for the quasiparticle energy $\varepsilon_{B}(\mathbf{p})$ is changed for $E(\mathbf{p})$ defined by (5) ( $\omega_{0}$ in the notation of [4] with exactly the same definition),

$$
V_{B}^{(1)}\left(\mathbf{p}, e_{p}\right) \longrightarrow V_{B}^{(1)}(\mathbf{p}, E(\mathbf{p}))
$$

the binding energy $E$ turns to be equal

$$
E_{B}=20.0 \mathrm{MeV} / \text { nucleon }
$$

The idea of the change (33) was considered in $[3,4]$ by the comparison of the formulas in the Brueckner theory and in the Green's function method with the diagram expansions for the interaction energy which differ in the two approaches. Equation (33) corresponds to several energy diagrams in the perturbation theory. But the solution of (5) for $E(\mathbf{p})$ corresponds to the completely renormalized energy when formally all diagrams of infinite series are taken into account. Thus, here, one has to be very careful with overcounting, the fact also mentioned in [4]. As a result, it is reasonable to compare the value of the binding energy in the spectral function method with its value in the Brueckner theory $E_{B}=$ 16.7 MeV/nucleon but not $E_{B}=20.0 \mathrm{MeV} /$ nucleon. In this comparison, formula (17) for the approximation $a_{i}$ is to be used.

Compare the expressions (13) and (14) with the values of $E_{\mathrm{QC}}$ and $E_{\mathrm{QP}}$ to get the normalizing factor $Z$ :

$$
Z=\frac{E_{\mathrm{QC}}}{E_{\mathrm{QP}}}=0.66 \text {, }
$$

which consequently gives $Z^{2}=0.44$.

Compare the expressions (14) and (15) with the values of $E_{\mathrm{QP}}$ and $E_{\mathrm{EQP}}$ to find the contribution $\Delta E$ of the second term in the right side of (15) to the value of the binding energy:

$$
\Delta E=E_{\mathrm{EQP}}-E_{\mathrm{QP}}=1.5 \mathrm{MeV} / \text { nucleon. }
$$

This corresponds to the contribution $\Delta E_{1}=Z^{2} \Delta E$ of the second term in the right side of (17) to the value of the binding energy:

$$
\Delta E_{1}=0.66 \mathrm{MeV} / \text { nucleon }
$$

Finally, the binding energy $E_{i}$ corresponding to the approximation (17) to the spectral function $a_{i}$ turns to be equal

$$
E_{i}=E_{\mathrm{QP}}+\Delta E_{1}=16.56 \mathrm{MeV} / \text { nucleon, }
$$

which is very close to the value $E B=16.7 \mathrm{MeV} /$ nucleon in the Brueckner theory.

\section{Conclusions}

Self-consistent approximations used in the Brueckner theory and in the Green's function method lead to almost coinciding values of the binding energy provided the correct formula (17) for the spectral function is used. The Brueckner formalism as well as its applications is found in many publications using various approaches of presentation [4]. This theory is strictly applicable to zero-temperature case. On the contrary, the Green's function method in Kadanoff-Baym version has certain advantage going beyond these restrictions. As mentioned in [6], "it can be now conclusively stated that selfconsistent spectral functions should (and perhaps equally important now can) be included in any serious consideration of nuclear properties being it in ground state, excited state, or in a state of nonequilibrium." For the study of nonequilibrium phenomena, the method can be used with constraints to define an initial condition. It should be stated that, in the case of nonequilibrium phenomena, different forms of the Kadanoff-Baym equations can be preferable for different physical systems $[18,19]$.

\section{References}

[1] L. P. Kadanoff and G. Baym, Quantum Statistical Mechanics, Benjamin, New York, NY, USA, 1962.

[2] L. P. Kadanoff and G. Baym, Quantum Statistical Mechanics, Perseus Books, Cambridge, Mass, USA, 1989.

[3] H. S. Köhler, "Spectral functions in nuclear matter," Physical Review C, vol. 46, no. 5, pp. 1687-1696, 1992.

[4] H. S. Köhler and R. Malfliet, "Extended quasiparticle approximation and Brueckner theory," Physical Review C, vol. 48, no. 3, pp. 1034-1045, 1993.

[5] H. S. Köhler and K. Morawetz, "Correlations in many-body systems with two-time Green's functions," Physical Review C, vol. 64, no. 2, Article ID 024613, 2001.

[6] H. S. Köhler, "Beyond the quasi-particle picture in nuclear matter calculations using Green's function techniques," Journal of Physics, vol. 35, article 384, 2006.

[7] W. D. Kraeft, D. Kremp, W. Ebeling, and G. Ropke, Quantum Statistics of Charged Particle Systems, Springer, Berlin, Germany, 1986.

[8] V. Spicka and P. Lipavsky, "Quasiparticle Boltzmann equation in semiconductors," Physical Review Letters, vol. 73, no. 25, pp. 3439-3442, 1994.

[9] V. Spicka and P. Lipavsky, "Quasiparticle Boltzmann equation in semiconductors," Physical Review B, vol. 52, no. 20, pp. 1461514635, 1995.

[10] M. Arshad, A. S. Kondratyev, and I. Siddique, "Spectral function and kinetic equation for a normal Fermi liquid," Physical Review $B$, vol. 76, no. 5, Article ID 54306, 2007.

[11] E. C. Titchmarsh, Introduction to the Theory of Fourier Integrals, Clarendon, Oxford, UK, 1975.

[12] M. J. Lighthill, Introduction to Fourier Analysis and Generalized Functions, Cambridge University Press, Cambridge, UK, 1960.

[13] Y. A. Brychkov and A. P. Prudnikov, Integral Transform of Generalized Functions, Gordon and Breach, New York, NY, USA, 1989.

[14] W. Appel, Mathematics for Physics and Physisists, Princeton University Press, Princeton, NJ, USA; Oxford, UK, 2007. 
[15] G. B. Arfken and H. J. Weber, Mathematical Methods For Physisists, Academic Press; Elsevier, 2005.

[16] K. A. Brueckner and D. T. Goldman, "Single particle energies in the theory of nuclear matter," Physical Review, vol. 117, no. 1, pp. 207-213, 1960.

[17] H. S. Köhler, “Theory of finite nuclei,” Physical Review, vol. 137, no. 5, pp. B1145-B1157, 1965.

[18] A. S. Kondratyev and I. Siddique, "Kinetic equations in the theory of normal Fermi liquid," Low Temperature Physics, vol. 34, no. 2, pp. 137-141, 2008.

[19] A. S. Kondratyev and N. Shahid, "Different forms of the Kadanoff-Baym equations in quantum statistical mechanics," Low Temperature Physics, vol. 37, article 777, no. 10, 2011. 

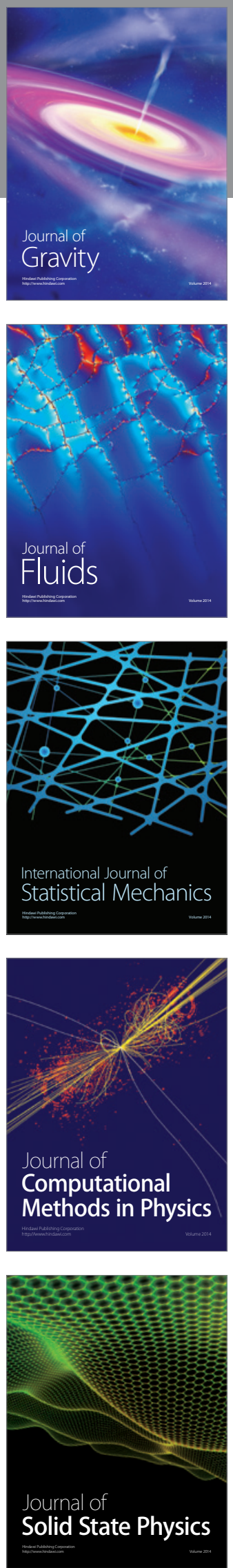

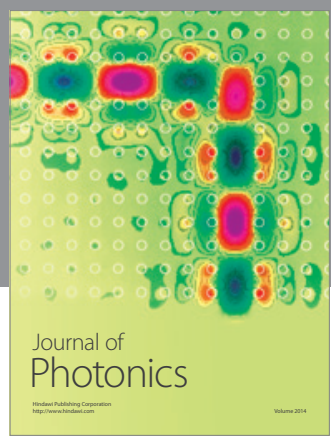

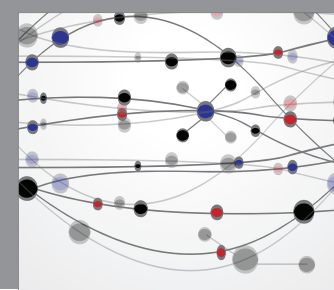

The Scientific World Journal

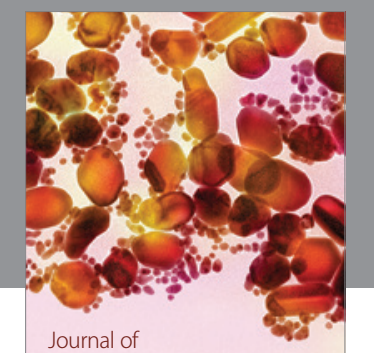

Soft Matter
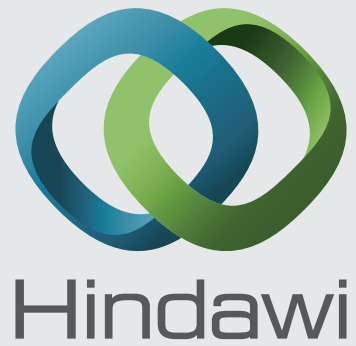

Submit your manuscripts at

http://www.hindawi.com
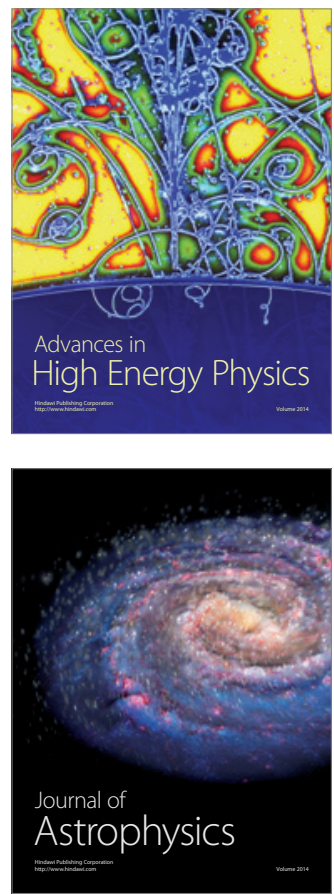
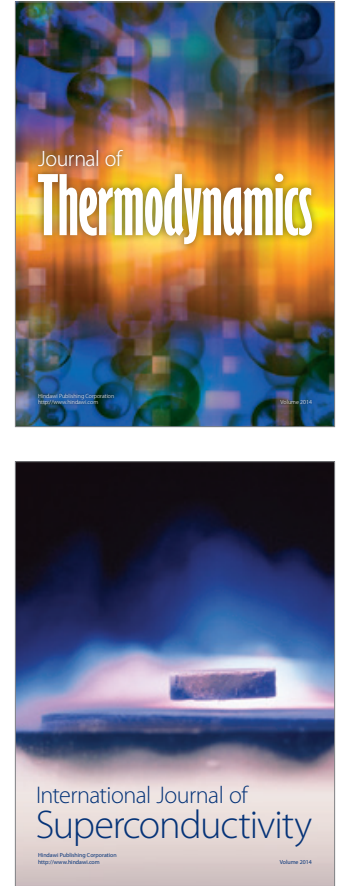
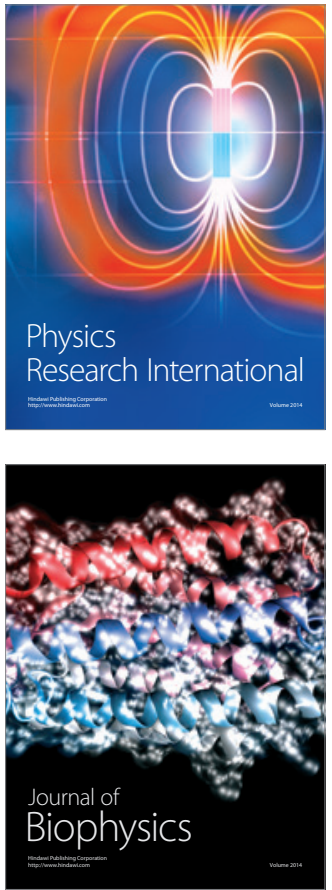
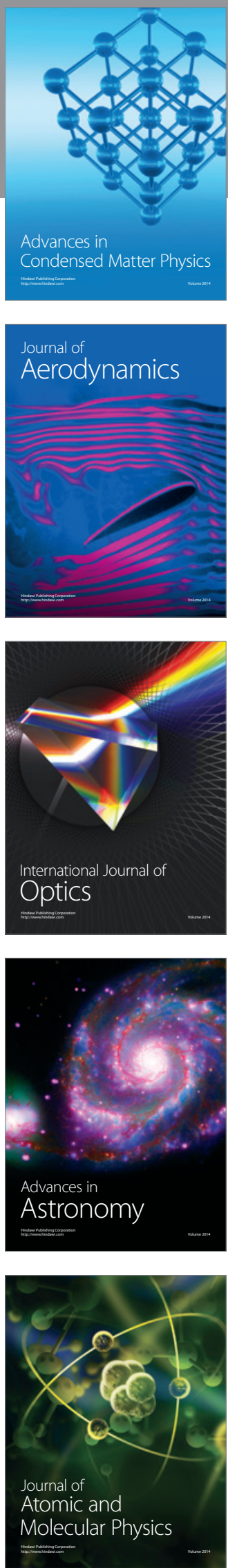